\title{
Highly-efficient flat-optics inverse design platform via fast trained neural predictors
}

\author{
Maksim Makarenko ${ }^{\dagger, *, 1}$, Arturo Burguete-Lopez ${ }^{*, 1}$, Fedor Getman ${ }^{*, 1}$, Andrea \\ Fratalocchi $^{1}$ \\ ${ }^{1}$ PRIMALIGHT, Faculty of Electrical Engineering; Applied Mathematics and Computational Science, King \\ Abdullah University of Science and Technology, Thuwal 23955-6900, Saudi Arabia \\ * These authors contributed equally to this work. \\ †corresponding author, E-mail: maksim.makarenko@kaust.edu.sa
}

\begin{abstract}
We introduce a universal design platform for the development of highlyefficient wavefront engineering structures. To validate this methodology, we fabricated many different optical devices with an experimental efficiency exceeding 99\%. (C) 2021 The Author(s)
\end{abstract}

\begin{abstract}
Main Text
Introduction

The production of compact, portable, and efficient optical devices is presently hindered by the bulky, expensive, and hard to manufacture nature of traditional optics [1,2]. Flat-optics, represented by surface structures composed of sub-wavelength spaced nanoscale elements, offer the possibility to replace conventional optics with highly integrated and versatile flat elements [2,3]. Despite the extensive work published in this field, commercial applications in the visible are still hindered by limited efficiencies, mainly due to absorbing losses in flat-optics elements from few hundreds of nanometers to microns. In this work, we propose a patented [4] flat optics design platform that addresses these issues by merging concepts from evolutionary algorithms to deep learning with convolutional neural networks (CNN). This platform exploits a previously unrecognized aspect of dielectric nanoresonators, namely the existence of a physical layer that acts as a feed-forward neural network that can approximate any user-defined function. With this approach, flat-optics structures can reach thicknesses as low as $50 \mathrm{~nm}$, and become almost lossless at any propagating frequency.
\end{abstract}

\section{Methods}

Inverse design of such ultra-thin flat-optics structures is carried out by an Autonomous Learning Framework for Rule-based Evolutionary Design (ALFRED) [5]. An optimized search for the desired resonance's configuration is based on a particle swarm optimizer (PSO) [6], coded in parallel to run on supercomputing architectures. The swarm performs a parallel communicative search on a randomly distributed particle array (Fig. 1a, bee), with each particle being a particular geometry that can solve the problem. The main bottleneck to this optimizer is the time required for each particle to evaluate the cost function's value. In this computation, each particle needs to obtain the structure's output response through costly first-principle simulations. In our problem, we usually optimize tens of different degrees of freedom and the resulting Finite-Difference Time-Domain (FDTD) time is around hundreds of thousands of core hours for a single structure. For this reason, we developed a neural network predictor unit attached to each particle (Fig. 1a), which predicts the outcome of FDTD simulations. The predictor network takes an array of single units as input and maps it to transmission/reflection/phase spectra. This network consists of two main parts: an encoder, presented by a convolutional neural network (CNN), which transforms an input image space to a low-dimensional feature representation, and a decoder, a group of fully-connected networks (FCN) connected to a feature space through a non-trainable optical switch, mapping a feature space to spectral points.

\section{Results}

We will discuss the implementation and characterization of different highly efficient flat-optics devices, including polarizer beam splitters at different wavelengths with bandwidths covering the whole visible, polarizing efficiency exceeding 99\%, and extinction ratio over 10000, multiband dichroic mirrors with efficiency around 90\% (Fig. 1b) and polarization-dependent color filters (Fig. 1c) that can support a novel type of display technology based on two sub-pixels instead of three as in traditional RGB screens. 


\section{References}

1. W. T. Chen, A. Y. Zhu, V. Sanjeev, M. Khorasaninejad, Z. Shi, E. Lee, and F. Capasso, "A broadband achromatic metalens for focusing and imaging in the visible," Nat. Nanotechnol. 13, 220-226.

2. Q. He, S. Sun, S. Xiao, and L. Zhou, "High-efficiency metasurfaces: Principles, realizations, and applications," Adv. Opt. Mater. 6, 1800415.

3. S. Shrestha, A. C. Overvig, M. Lu, A. Stein, and N. Yu, "Broadband achromatic dielectric metalenses," Light. Sci. \& Appl. 7, 85 .

4. A. Fratalocchi, F. Getman, M. Makarenko, and A. Burguete-Lopez, "Light processing device based on multilayer nanoelements," (US Patent Application No. 62/844,416).

5. F. Getman, M. Makarenko, A. Burguete-Lopez, and A. Fratalocchi, "Broadband vectorial ultra-flat optics with experimental efficiency up to $99 \%$ in the visible via universal approximators," Light. Sci. Appl. (2021, to appear).

6. J. F. Kennedy, R. C. Eberhart, and Y. Shi, Swarm Intelligence, The Morgan Kaufmann Series in Evolutionary Computation (Morgan Kaufmann Publishers).

Acknowledgments This research received funding from KAUST (Award OSR-2016-CRG5-2995). Parallel simulations are performed on KAUST's Shaheen supercomputer.

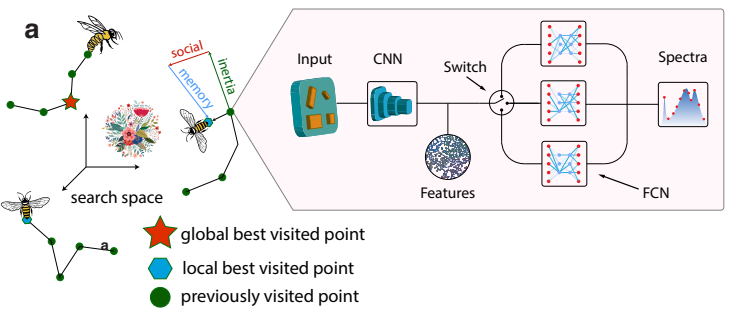

c



Fig. 1. (a) Predictor and optimizer units of ALFRED. (b) Comparison between a simulated and measured performance for the produced dichroic mirror structure (left) with Scanning-Electron Microscope (SEM) picture (right). (c) Polarization-dependent color filters with the corresponding SEM images (right). 